\title{
Moisture Proof Columnar Cesium Iodide (CsI) Layers for Gas Avalanche Microdetectors
}

\author{
I.J.Park ${ }^{1}$, H.S.Cho ${ }^{1}$, W.S. Hong ${ }^{2}$, V.Perez-Mendez ${ }^{1}$, J.Kadyk ${ }^{1}$ \\ ${ }^{1}$ Physics Division, ${ }^{2}$ Engineering Division, Lawrence Berkeley National \\ Laboratory, Berkeley, CA 94720
}

\begin{abstract}
Cesium iodide columnar layers having a diameter of $\approx 3$ $\mu \mathrm{m}$, and wall spacing of $\approx 1 \mu \mathrm{m}$ act as secondary electron emitters and can be used for detection of radiation: charged particles, X-rays and gamma rays. With a large enough electric field across the columnar layers, $\approx 400 \mu \mathrm{m}$ in thickness, gas avalanche gain is evident when placed in a suitable gas, such as P10 or argon-ethane mixtures. The cesium iodide columns are damaged by ambient moisture. This damage can be prevented by evaporating protective layers of insoluble, low boiling point inorganic materials, such as mercuric iodide. Columnar layers with $20 \mathrm{~nm}$ coatings of mercuric iodide yield more than 30,000 electrons on average when traversed by electrons from a ${ }^{90} \mathrm{Sr}$ beta source.
\end{abstract}

\section{INTRODUCTION}

We have previously made scintillating $\mathrm{CsI}(\mathrm{Tl})$ columnar layers for use in X-ray medical imaging pixel detectors[1]. We have also used CsI columnar layers for secondary electron emission from the column walls, followed by gas avalanches in the space between columns. The enhanced electron signal is then coupled to gas avalanche microdetectors, and the concentration of electron production to a small interaction region allows better localization of incident particles, and better detection efficiency, both independent of incident angle, as well as improved time resolution[2].

In this paper we will describe characteristics of CsI columnar layers coated with mercuric iodide as independent detectors operating in P10 or argon-ethane(50/50) gas mixtures. We describe the pulse height enhancement, timing response, and other characteristics.

\section{StRUCTURE}

The production of columnar CsI layers and their $\mathrm{HgI}_{2}$ coatings are shown in Fig 1. Fig. 1(a) shows the evaporation method for columnar CsI production. Pellets of CsI are fed into an evaporation boat. The enclosure is filled with argon at a pressure of 5 torr. The substrate for the CsI layer (e.g. ceramic, glass, aluminum, plastic) is held at a temperature of $120^{\circ} \mathrm{C}$. The combined effect of the rotation and evaporation in the argon atmosphere causes the CsI to grow in columns as described in[3]. As shown in Fig. 1(b), the columnar layer is then placed in a container with a weighed amount of $\mathrm{HgI}_{2}$, calculated to provide a uniform coating on the CsI columns. The container is heated for an hour at $350{ }^{\circ} \mathrm{C}$ and then cooled slowly.

This work was supported by the Director, Office of Energy Research of the U.S. Department of Energy Contract No. DEAC03-96SF00098
Fig. 2 shows the scanning electron microscope (SEM) photographs of the coated columnar CsI layers. The column diameter was measured to be $3-6 \mu \mathrm{m}$ and the wall spacing was 1-2 $\mu \mathrm{m}$. The columns show a well-defined geometry, which permits the development of gas avalanches within the columnar spacing.

(a)

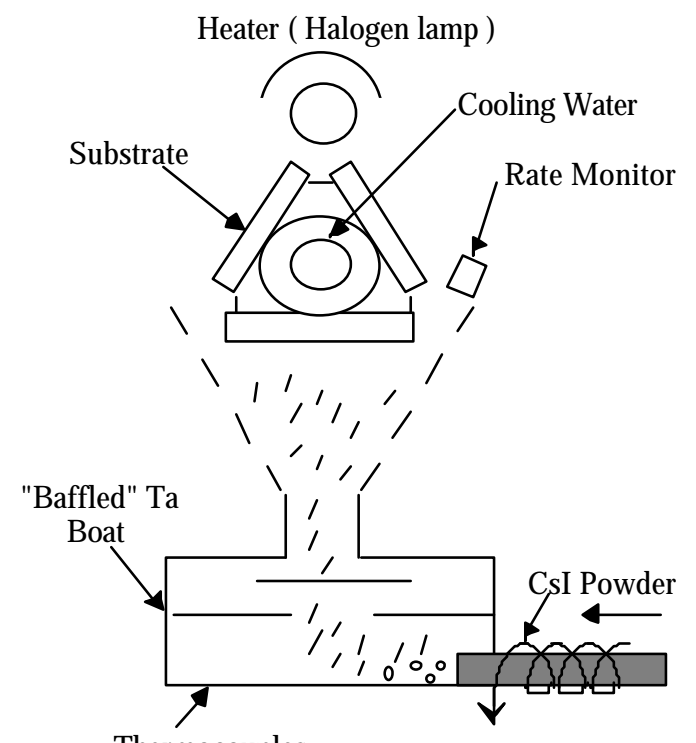

Thermocouples

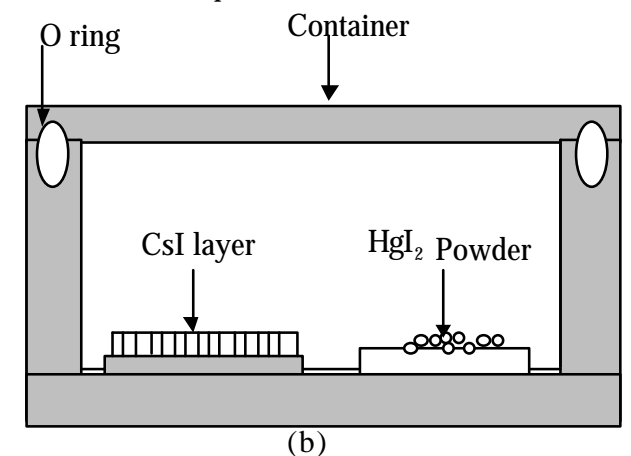

Fig. 1. (a) Evaporation method for producing the columnar CsI layer and (b) Method of coating the CsI columns with $\mathrm{HgI}_{2}$

CsI has long been recognized as a secondary electron emitter (SEE). The SEE yield for charged particle traversal has been measured for flat layers[4,5] and for porous CsI material[6]. 


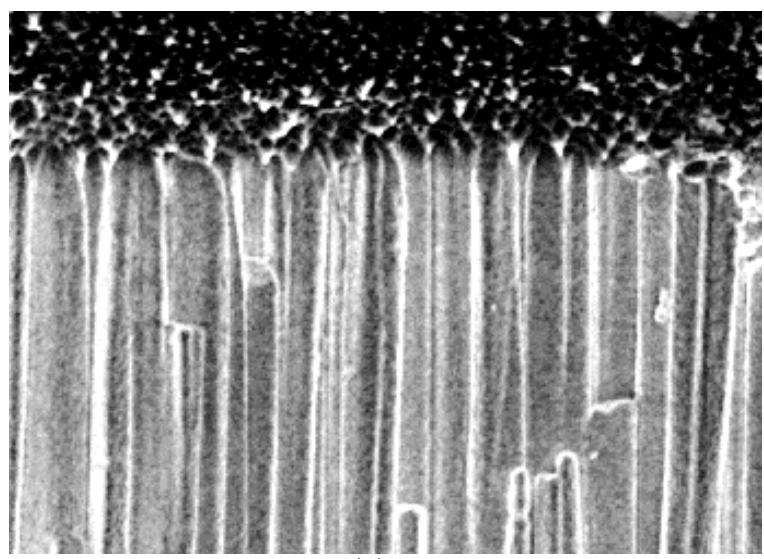

(a)

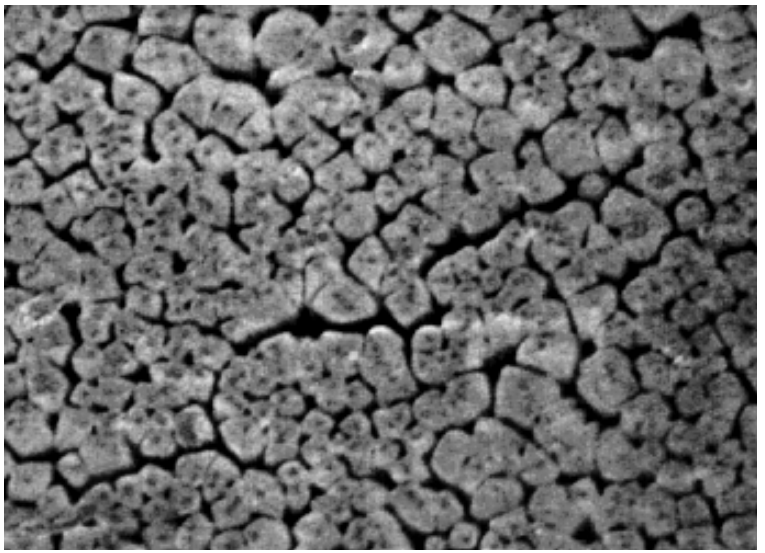

(b)

Fig. 2. Scanning electron microscope (SEM) photographs of $350 \mu \mathrm{m}$ thick columnar CsI layer (a) from the side view and (b) from the top view.

It has been recognized that the yield of SEE for a single layer traversal is small, but that multiple traversals, as in porous CsI and in our columnar material, give a larger yield. Our columnar layer has a relatively well-defined geometry compared to a porous layer and, as will be shown below, produces gas avalanches, which increase the signal amplitude.

\section{PERFORMANCE AND CHARACTERISTICS}

We have fabricated CsI columnar layers 300-400 $\mu \mathrm{m}$ thickness and coated them with $\mathrm{HgI}_{2}$ of approximately $20 \mathrm{~nm}$ thickness. Initially we coupled a CsI layer to a conventional micro strip gas chamber (MSGC). A grid served both as a drift electrode for the MSGC and permitted electrons produced in the CsI layer to enter the sensitive region of the MSGC chamber as shown in Fig 3(a).

The increase in signal from the MSGC is shown in Fig 3(b). The signal enhancement factor in fig. 3(b) is the ratio of the signal seen in the MSGC at a voltage, $\mathrm{V}_{\text {csi }}$, across the CsI layer to the signal due to ions formed solely in the gas gap (i.e. with $\mathrm{V}_{\mathrm{csi}}=0 \mathrm{~V}$ ). As can be seen, a large amount of electron amplification occurs within the activated CsI layers, whether uncoated, or coated with $\mathrm{HgI}_{2}$.
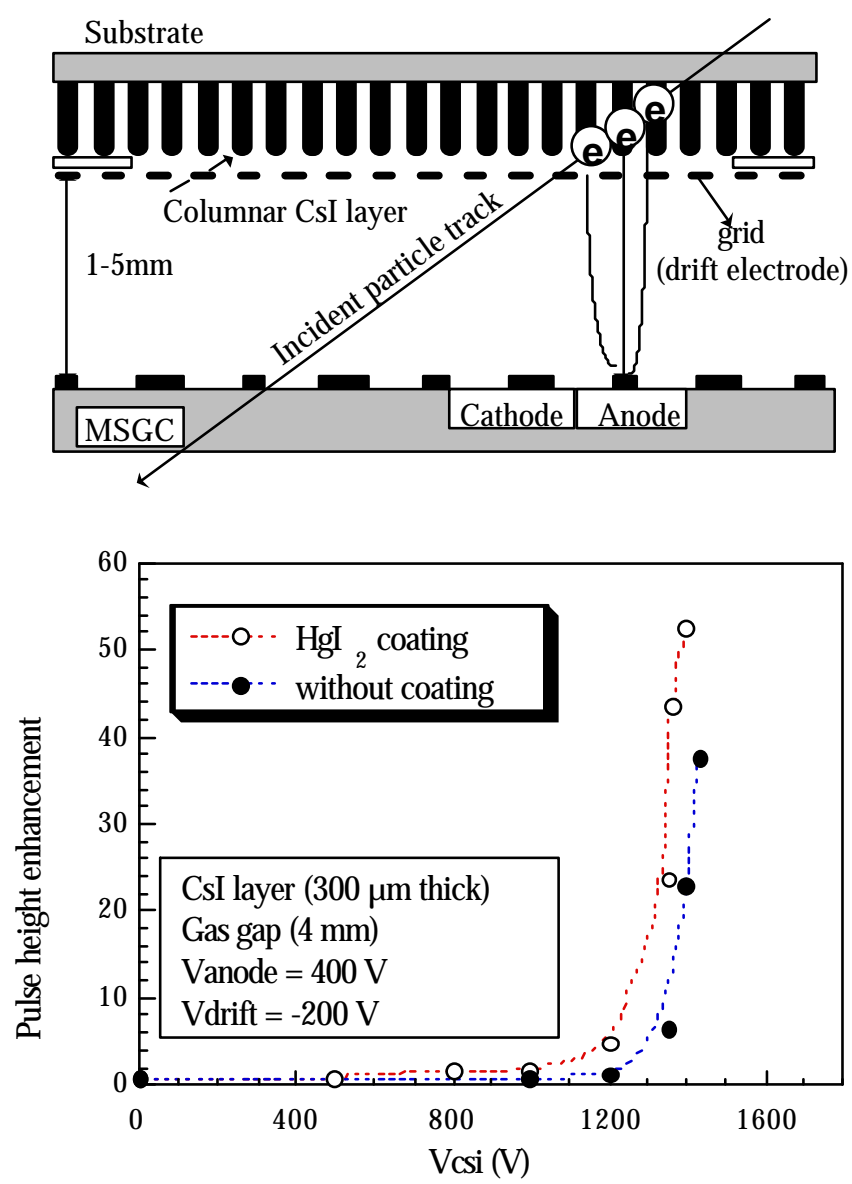

(a)

(b)

Fig. 3. (a) Mounting of a columnar CsI layer on drift plane of MSGC (b) Increased signals from MSGC coupled to the $\mathrm{HgI}_{2}$ coated columnar CsI layer and uncoated columnar CsI layer ( $\mathrm{V}_{\text {anode }}$ is anode voltage and $\mathrm{V}_{\text {drift }}$ is drift voltage of MSGC)

Subsequently we mounted the $\mathrm{HgI}_{2}$ coated $\mathrm{CsI}$ layer independently of the MSGC and collected electrons from the avalanches in the CsI layer on the signal collection grid, as indicated in Fig. 4(a). We used electrons $(>1 \mathrm{MeV})$ from a ${ }^{90} \mathrm{Sr}$ beta source. This collection plane can be replaced with pixel pads with electronics readout, as described in ref.[7] or parallel strips as in MSGC according to application field.

With the grid at a positive potential relative to the CsI, avalanche electrons are collected, yielding the distributions shown in Fig. 4(b) with noise spectrum. Signals from columnar CsI layer was large enough and clearly distinguishable to noise. With a negative bias on the grid, positive ions are collected. The yielding a distribution is similar in magnitude to that of the electrons, as shown in Fig. 4(c). This helps to confirm the assumption that in addition to starting the signal by secondary electron emission from the CsI surface, avalanche multiplication between the columns and in the grid space also occurs.

Therefore, an additional device, such as an MSGC, may not be needed to achieve the desired signal level, thus simplifying the detector structure. 


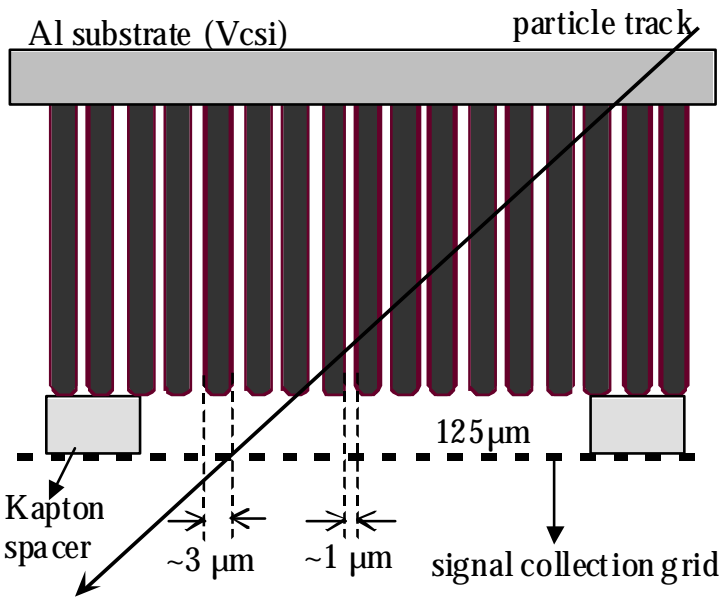

(a)

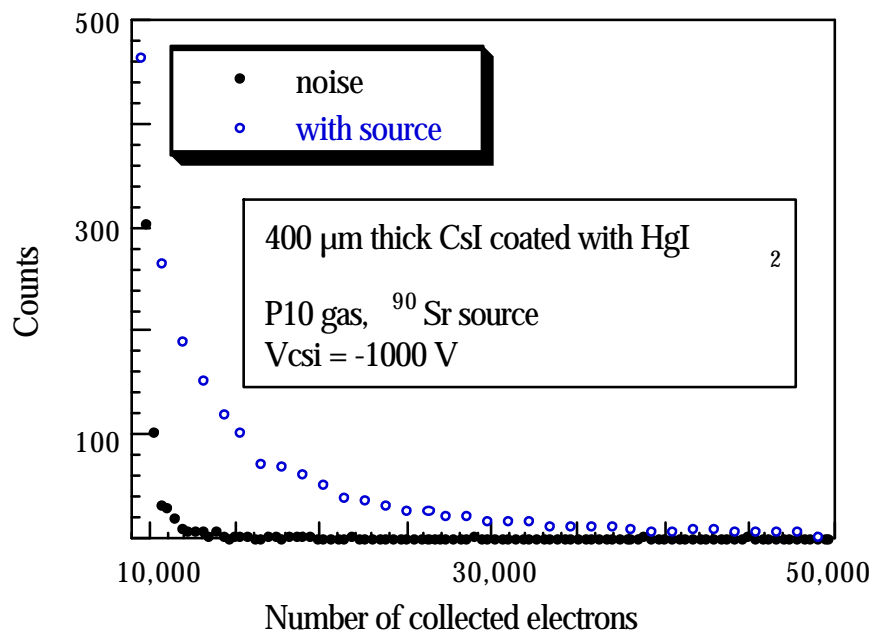

(b)

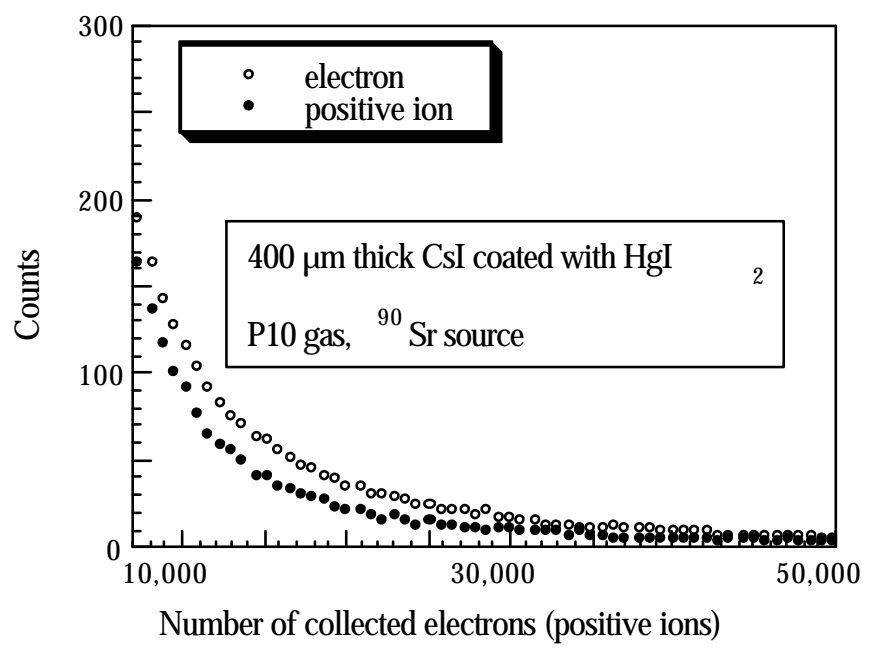

(c)

Fig. 4. (a) Columnar CsI layer coupled to grid anode (b) Pulse height spectra from ${ }^{90} \mathrm{Sr}$ source - electron collection (c) Pulse height spectra - positive ion collection

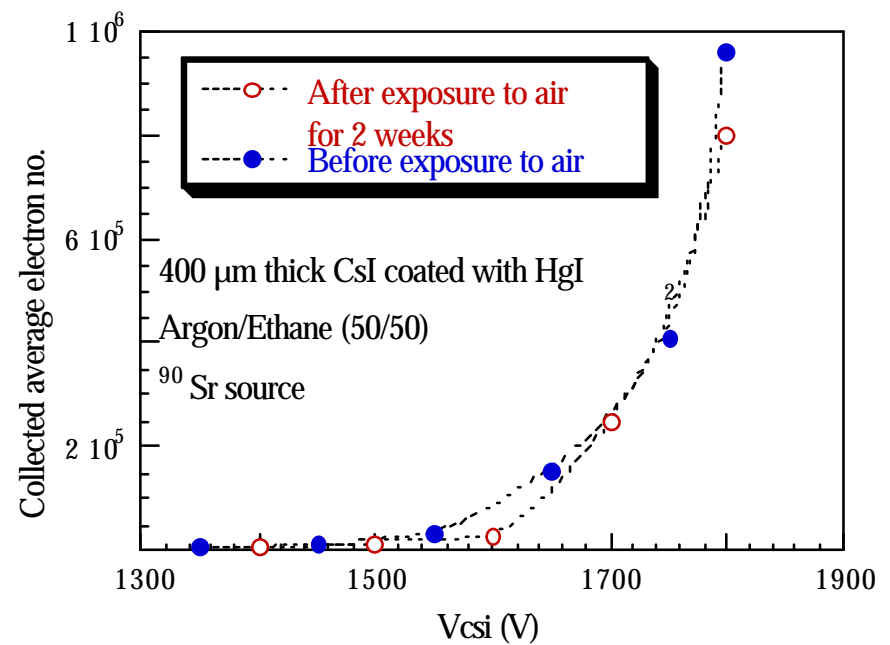

Fig. 5. Number of collected electrons for before and after exposure to air as a function of $\mathrm{V}_{\mathrm{csi}}$.

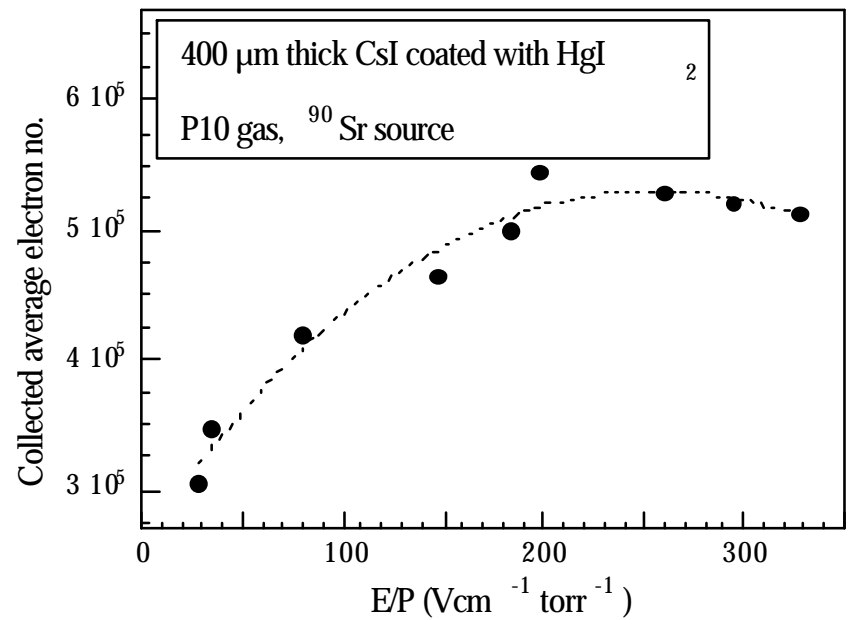

Fig. 6. Collected charge at different gas pressure as function of electric field per unit pressure $(\mathrm{E} / \mathrm{P})$

We measured average collected electron numbers from CsI coated with $\mathrm{HgI}_{2}$ before and after two weeks exp osure to air as function of $\mathrm{V}_{\mathrm{csi}}$. In this measurement, we used argon/ethane(50/50) gas mixtures and ${ }^{90} \mathrm{Sr}$ beta source. Fig. 5 shows the results. For $\mathrm{HgI}_{2}$ protected layers, there was no significant change in the average collected electron number observed after exposure to air. Therefore we confirm $\mathrm{HgI}_{2}$ coating is an effective protecting layer preventing moisture damage.

We also measured the gas avalanche gain at reduced gas pressures and the result is shown in Fig. 6. For a gas avalanche detector, gas gain is dependent on the mean free path of electrons produced in the gas media and on the electric field. When the gas pressure is reduced, the gain is increased. This is as expected, since electrons gain more energy between collisions at reduced pressure, enhancing the probability of ionization. We believe that since the columnar structure dimensions are comparable to the collision mean path, the curve does not exhibit the usual "exponential" shape.

All of these results are consistent with our assumption of gas avalanches occurring between columns, thus the columnar 
CsI layer can be defined as a secondary emission (SE) gaseous detector.

\section{TIMING RESPONSE}

For particle detectors, fast timing is often important. Using a ${ }^{90} \mathrm{Sr}$ source, we measured the timing resolution of the columnar CsI secondary emission (SE) gaseous detector. The measurement system is shown in Fig. 7(a). In this system, timing filter amplifiers (TFA) were used for fast shaping time. The signals from each TFA were connected to constant fraction discriminators (CFD) for accurate timing and the CFD signals were used as the start and stop signals in a time to amplitude converter. A gate \& delay generator converts the amplifier signal to a logic signal, for the pulse height analyzer gate. The results are shown in Fig. 7(b) with a gaussian fitting curve. From this, the timing resolution for one columnar CsI SE gaseous detector was determined to $7.8 \mathrm{~ns} \mathrm{rms}$.

(a)

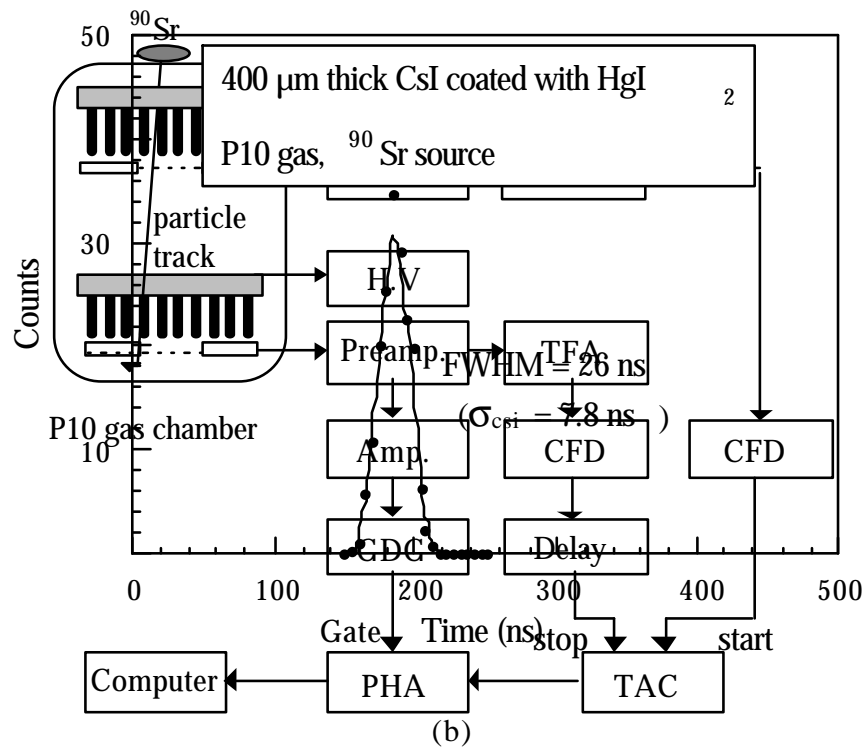

Fig. 7. (a) Timing measurement system composed with two columnar CsI SE gaseous detectors (b) Timing spectrum with a gaussian fitting curve.

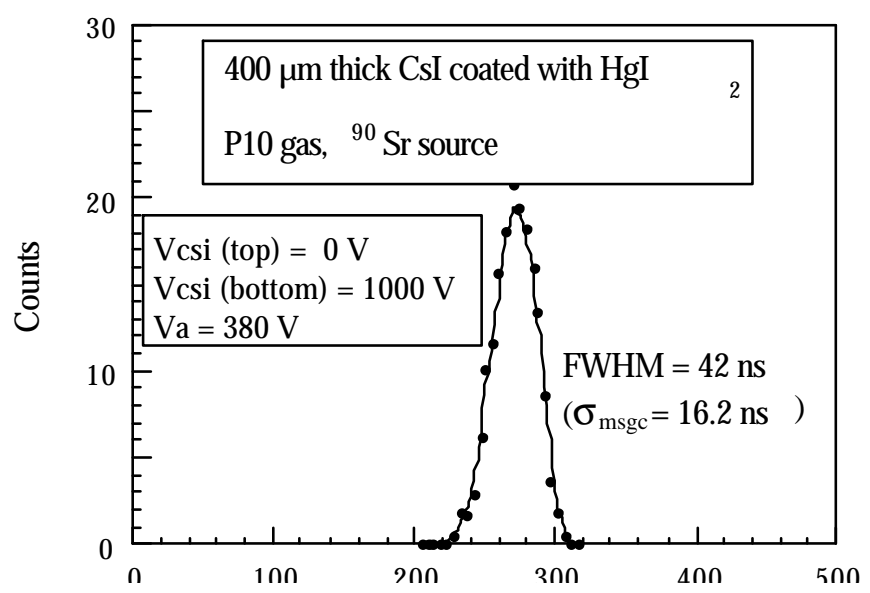

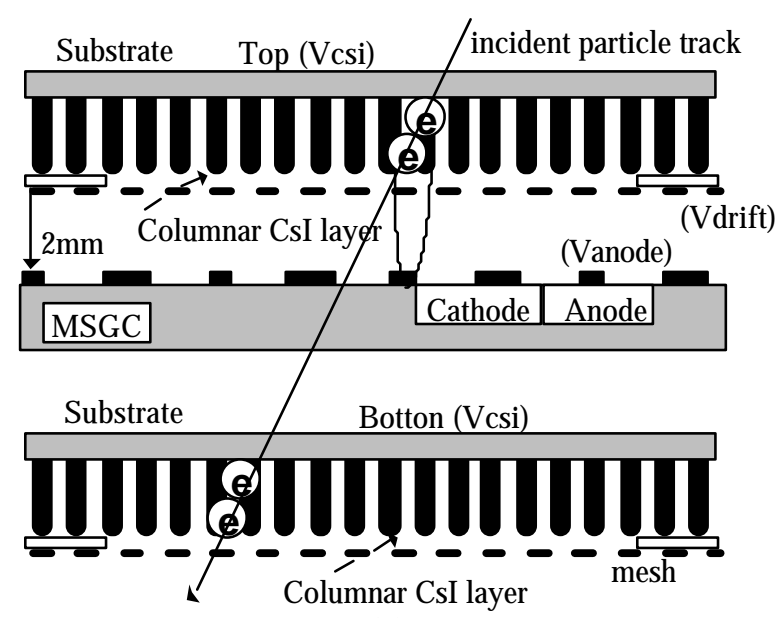

(a)

(b)

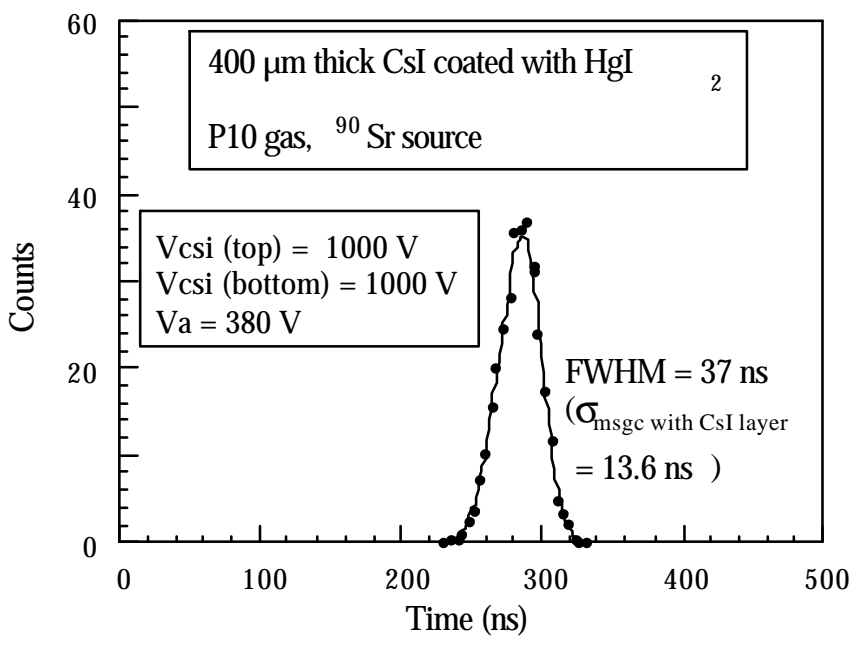

(c)

Fig. 8. Timing of MSGC when CsI is used as the drift plane. (a) the schematic of measurement system (b) Timing spectrum when top CsI was not activated (c) Timing when top CsI was activated.

To identify the effect of a columnar CsI layer on the timing of a conventional MSGC, we mounted one CsI layer on the drift plane of the MSGC and the other CsI layer was used as the start pulse of the timing measurement. Fig. 8(a) shows schematically the experimental setup. Fig. 8(b) is the timing spectrum when the top CsI was not activated, i.e., $\Delta \mathrm{V}_{\mathrm{csi}}=0 \mathrm{~V}$, and Fig. 8(c) shows the timing when $\Delta \mathrm{V}_{\mathrm{csi}}$ was $1000 \mathrm{~V}$. In this measurement, we applied $V_{d}=-1000 \mathrm{~V}$ to the top drift plane, and $\mathrm{V}_{\mathrm{a}}=+380 \mathrm{~V}$ to the MSGC anode. We deconvolved the contribution of the CsI start pulse from the timing spectrum. Timing of the conventional MSGC alone was $16.2 \mathrm{~ns} \mathrm{rms}$, and MSGC coupled to CsI layer was $13.6 \mathrm{~ns}$ rms. As expected, the CsI layer improves timing of the coupled MSGC, though not by a large factor.

\section{X-RAY AND $\gamma$-RAY SENSITIVITY}

We exposed the CsI layer to $60 \mathrm{keV}$ gamma-rays from an ${ }^{241} \mathrm{Am}$ source and to X-rays from a $19 \mathrm{kVp}$ generator. The noise spectrum was also measured by removing the source from detector, and this was subtracted from the ${ }^{241} \mathrm{Am}$ spectrum. The subtracted spectrum is shown in fig. 9(a). The 
gas amplification starts at the interaction position and is initiated by emission of secondary electrons from the CsI

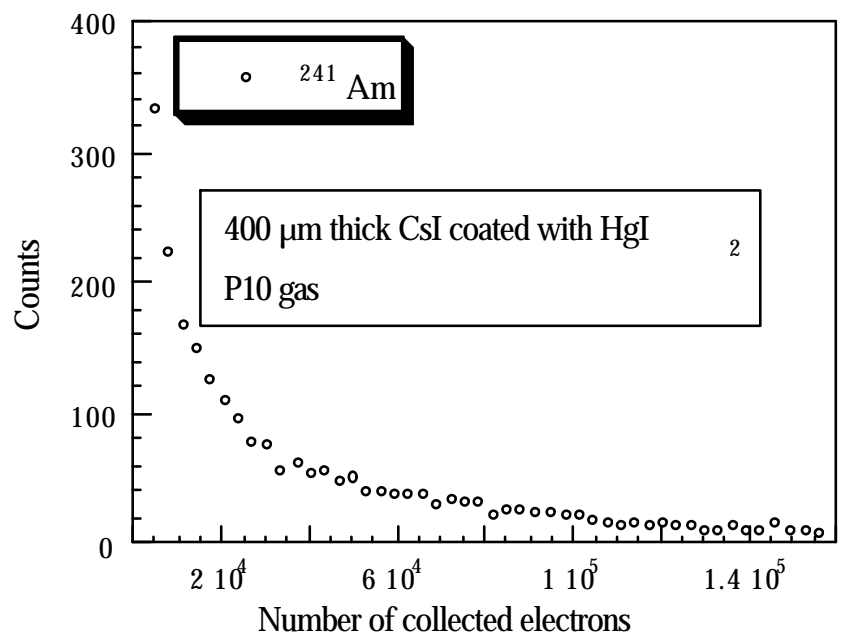

surface.

(a)

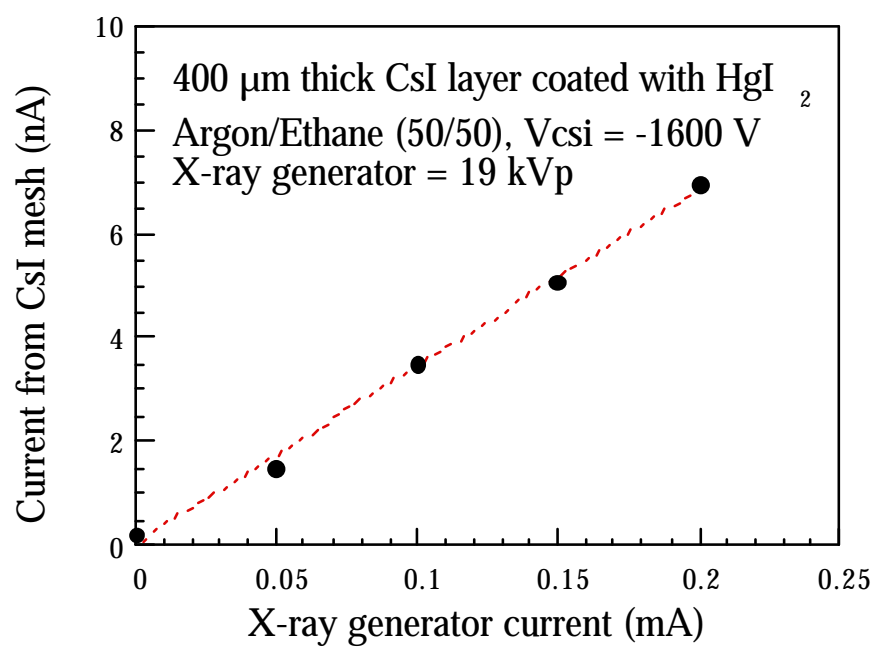

(b)

Fig. 9. (a) ${ }^{241} \mathrm{Am} 60 \mathrm{keV}$ gamma-ray spectrum (b) Current signal from steady state $\mathrm{X}$-ray exposure.

The linearity between X-ray intensity and signal response is shown in Fig. 9(b). This result presents the possibility to use the columnar CsI SE gaseous detector for X-ray radiography. For this application, the efficiency and spatial resolution for various photon energies has to be investigated in detail.

Since these energies straddle the mean energies used in diagnostic X-ray imaging these data show that these layers can be used for digital radiography when pixel detector pads as mentioned in reference[7] are used as the detector element in place of a grid anode.

\section{SUMMARY AND CONCLUSIONS}

CsI columnar layers 300-400 $\mu \mathrm{m}$ thick when placed in electric fields of $30-50 \mathrm{kV} / \mathrm{cm}$ produce large signals in response to charged particles, gamma-rays and X-rays. The mechanism of signal enhancement is believed to be a combination of secondary electron emission from the columnar surfaces and gas avalanches induced by the SEE electrons in the space between columns. The average signal is $>30,000$ electrons per incident ${ }^{90} \mathrm{Sr}$ beta particle. The timing resolution is $7.8 \mathrm{~ns}$ rms.

When used as a drift plane in conjunction with a gas avalanche micro detector such as MSGC, micro gap chamber (MGC), micro dot chamber (MDOT) and gas electron multiplier (GEM), the columnar CsI layer gives somewhat improved time resolution, and is expected to eliminate the angle dependence of space resolution and detection efficiency[2]. Moreover, sufficient signal is obtained from the CsI layer that, it is possible to use it as the detector by itself, without coupling it to an MSGC or other gas avalanche device, thus simplifying the entire detection system.

The ease and simplicity of making this device is another advantage. At present $\mathrm{CsI}(\mathrm{Tl})$ layers in sizes up to $50 \times 50 \mathrm{~cm}^{2}$ are made commercially for medical imaging such as digital radiography by various companies[8].

\section{ACKNOWLEDGMENTS}

We would like to thank Allan Lyon from the evaporation shop in LBNL for making the CsI columnar layers.

\section{REFERENCES}

[1] T. Jing et al., Nucl. Instr. and Meth., A368 (1996) 757.

[2] H. S. Cho et al., Nucl. Instr. And Meth., A422 (1999) 269.

[3] T. Jing., U. C. Thesis. LBNL. (May 1995).

[4] D. F. Anderson et al., Nucl. Instr. And Meth., A346 (1994) 102.

[5] A. Breskin et al., Nucl. Instr. And Meth., A345 (1994) 337.

[6] C. Chianelli et al., Nucl. Instr. And Meth., A273 (1998) 245.

[7] W. S. Hong et al., IEEE Trans. Nucl. Sci., NS44 (1997) 1001.

[8] For example EG\&G amorphous silicon group. Santa Clara, DPIX Palo Alto California. 